\title{
High Intensity Exercise Assessment of Special Populations
}

\author{
Julien S Baker*, Duncan Buchan, Lon Kilgore and Fergal Grace \\ Institute of Clinical Exercise and Health Science, School of Science, University of the West of Scotland, Hamilton, Lanarkshire, Scotland
}

Although originally used with able-bodied healthy subjects, high intensity cycle ergometry can be used in conjunction with specific populations to assess subjects with chronic disease or physical disability. The rationale for such an application has been that the factors limiting physical performance may be muscular or neurological in nature, rather than cardiorespiratory [1]. Therefore, testing their peripheral function may have diagnostic and prognostic value. However, important questions remain about the feasibility and reliability of high intensity cycle ergometry when subjects with a physical disability perform the test. Problems of standardisation arise because of the marked variation in ability, fitness levels and active muscle mass that may be independent of resistive force selection [1].

For example, many people with cerebral palsy cannot keep their feet on the pedals during the performance of high intensity cycle ergometry even when stirrups are used. However, these problems have been overcome and meaningful results have been obtained when the subjects had their feet taped to the pedals [2]. Further difficulties have been encountered in patients with extreme muscle weakness. These subjects on occasion find it impossible to complete a full pedal revolution. A mechanical solution to the problem was found by decreasing pedal crank length facilitating the rotation of the flywheel at a smaller pedal circumference. Although these problems are to a certain extent mechanistic/technical, selection of resistive forces that relate to active muscle tissue in these populations may be desirable. The greater mechanical resistance to motion inherent using resistive forces derived from total body mass (TBM) as opposed to fat free mass (FFM) may further compromise and confound the problems outlined.

For most healthy non-athletes, the assumption has been that the relationship between muscle mass and total body mass is similar. However, in certain segments of the population, i.e., those subjects that are obese, undernourished, have muscle atrophy, muscle hypertrophy, are amputees or have neuromuscular disease, this relationship may deviate from the normal. In these groups, the FFM may be smaller or greater than expected in relation to TBM. For these populations the assignment of a resistive force based on TBM may not only yield an overestimation/underestimation of maximal anaerobic performance, but may compromise further the health status of the patients themselves [3]. We have demonstrated significantly higher power outputs in our laboratory for able bodied subjects, when resistive forces reflect the lean tissue component of muscle mass as opposed to resistive force selection based on TBM [4].

The FFM protocol may also be an attractive alternative for the assessment of high intensity potential in the elderly population. This subject group may possess different lean tissue mass to fat mass ratios for reasons that may be medically related or non-pathological. The differences observed may be associated to issues that are to a certain extent, independent of health status such as social standing, depravation and emaciation. Optimal high intensity cycle ergometer resistive forces for this population is not known and guidelines for resistances used with healthy individuals are not applicable to geriatric subjects or patients with a disability-TBM to FFM ratio may be different when compared to able bodied populations. Further research is needed to pinpoint the optimal resistive force for subgroups including populations of intense interest such as children, overweight, underweight, elderly, and the disabled.
The biochemical and neural events associated with high intensity assessment are also worthy of study to facilitate a better understanding of the health issues relating to high intensity exercise ability. The development of the FFM protocol appears to be attractive in both the clinical and athletic evaluation of high intensity exercise performance in various subject populations. While this practical solution still requires validation, designing experiments to find optimal resistive forces based on FFM for the disabled will be difficult because of the wide spectrum of diseases and levels of residual ability found within and between these specific subject groups.

The task is not impossible, Van Mil et al. [3] has reported that an anthropometric estimate of lean tissue volume is a valid predictor of the optimal resistive force during a high intensity cycle ergometer test in both children and adolescents with neuromuscular disease. The findings of the study are encouraging and should contribute to a greater understanding of high intensity ability in similar populations and sub groups. The assessment of athletic ability that includes high intensity exercise assessment is warranted, particularly when we consider the performances associated with the current para olympics. It is crucial that as exercise scientists, we find valid and reliable means of evaluating the performances of these special athletes and clinical populations. This will provide them with valuable feedback in relation to performance indicators, and hopefully, provide a means to excel and improve upon the incredible athletic and lifestyle achievements that they have already obtained.

\section{References}

1. Inbar O, Bar-Or O, Skinner S (1996) The Wingate Anaerobic Test. Human Kinetics, Leeds.

2. Parker DF, Carriere L, Hebestreit H, Bar-Or O (1992) Anaerobic endurance and peak muscle power in children with spastic cerebral palsy. Am J Dis Child 146: 1069-1073.

3. Van mil E, Schoeber N, Calvert RE, Bar-or O (1996) Optimization of force in the Wingate Test for children with a neuromuscular disease. Med Sci Sports Exerc 28: 1087-1092.

4. Baker JS, Bailey DM, Davies B (2001) The relationship between total-body mass, fat-free mass and cycle ergometry power components during 20 seconds of maximal exercise. J Sci Med Sport 4: 1-9.

*Corresponding author: Julien S Baker, Institute of Clinical Exercise and Health Science, School of Science, Faculty of Science and Technology, University of the West of Scotland, Hamilton, Lanarkshire, Scotland, ML3 OJB, E-mail: jsbaker@uws.ac.uk

Received September 05, 2012; Accepted September 05, 2012; Published September 10, 2012

Citation: Baker JS, Buchan D, Kilgore L, Grace F (2012) High Intensity Exercise Assessment of Special Populations. J Sports Med Doping Stud 2:e123. doi:10.4172/2161-0673.1000e123

Copyright: @ 2012 Baker JS, et al. This is an open-access article distributed under the terms of the Creative Commons Attribution License, which permits unrestricted use, distribution, and reproduction in any medium, provided the original author and source are credited. 